\title{
Manipulating neurons with magnetogenetics
}

The Magneto channel, an orthogonal tool for optogenetic actuators, provides magnetic control over neuronal activity.

Optogenetic and chemogenetic actuators are widely used to manipulate neuronal activity in behaving animals. "While using the chemogenetic and optogenetic tools, we have acutely become aware of the fact that there are limitations," says Ali Güler from the University of Virginia in Charlottesville. These include the need for illumination in the case of optogenetics, which might interfere with natural behaviors, and slow kinetics in the case of chemogenetics.

To overcome these potential limitations, Güler and his team members and collaborators developed Magneto, a fusion of the calcium channel TRPV4 and ferritin, which mediates calcium influx into cells upon magnetic stimulation. The tool is small enough to be packaged into a single viral vector, which facilitates its application in the mouse and is an advantage over related, multicomponent tools. The researchers demonstrated that Magneto can induce action potentials in mouse organotypic brain slices and exert control over escape behaviors in zebrafish and place preference in mice.

Developing Magneto was a tour de force. Ion channels are notoriously difficult to manipulate and to express, as reflected by the fact that the team needed to test 21 constructs before arriving at Magneto. The researchers' persistence was rewarded when they observed the first Magneto-induced action potentials. “That was a big day," says Güler. The collaborative environment at the University of Virginia facilitated the further characterization of Magneto in slice cultures and animals, explains Güler. Students and postdocs from his and other labs started collaborating in a bottom-up approach and shared constructs before he became aware of them.

TRPV4 is widely expressed in the mouse and is sensitive to a variety of endogenous ligands, which could also act on Magneto, says Güler. Ideally, future versions of Magneto would be insensitive to endogenous ligands, to avoid unwanted activation of the channel. He also thinks that improving the sensitivity of Magneto will be helpful, as it would allow larger behavioral arenas to be used. Güler is actively working on additional magnetically controlled actuators to expand the magnetic toolbox.

Nina Vogt

\section{RESEARCH PAPERS}

Wheeler, M.A. et al. Genetically targeted magnetic control of the nervous system. Nat. Neurosci. http:// dx.doi.org/10.1038/nn.4265 (2016). 